\title{
The finite element method for ill-posed problems
}

RAIRO - Analyse numérique, tome 11, no 3 (1977), p. 271-278.

<http://www.numdam.org/item?id=M2AN_1977_11_3_271_0>

(C) AFCET, 1977, tous droits réservés.

L'accès aux archives de la revue « RAIRO - Analyse numérique » implique l'accord avec les conditions générales d'utilisation (http://www.numdam.org/ legal.php). Toute utilisation commerciale ou impression systématique est constitutive d'une infraction pénale. Toute copie ou impression de ce fichier doit contenir la présente mention de copyright.

\section{Numdam}

Article numérisé dans le cadre du programme

Numérisation de documents anciens mathématiques

http://www.numdam.org/ 


\title{
THE FINITE ELEMENT METHOD FOR ILL-POSED PROBLEMS ( $\left.{ }^{1}\right)$
}

\author{
by Frank NATTERER $\left({ }^{2}\right)$ \\ Communiqué par P G Ciarlet
}

Summary - The Tikhonov regularized solution is approximated by the finte element method $A$ relation between the regularization parameter and the mesh size is given which implies that the regularized solution and its fintte element approximation are of the same order of accuracy Applications are made to the Radon transform and numerical results are given for an integral equation of the first kind

\section{INTRODUCTION}

Assume that $E, F$ are real inner product spaces and $A: E \rightarrow F$ is a linear injectıve map. If $f \in$ range $(A)$, then there is a unique $y \in E$ such that

$$
A y=f \text {. }
$$

If $A^{-1}$ is unbounded, then the problem : Given $f$, find $y$, is called ill-posed. The reason for this is the following : If instead of $f$ only some $f_{\varepsilon}$ with $\left\|f-f_{\varepsilon}\right\|_{F} \leqslant \varepsilon$ is available, then the solution $\hat{y}_{\varepsilon}$ of $A \hat{y}_{\varepsilon}=f_{\varepsilon}$ (it at all existent) need not be close to $y$. But even if $f$ is known exactly, we still have the problem that any discrete version of $A$ is badly ill-conditioned. One of the standard remedies is to use more information on $y$. Let $V \subseteq E$ be a Hilbert space, the embedding $V \rightarrow E$ being compact. If we know that $y \in V$, then we can replace $\hat{y}_{\varepsilon}$ by the regularized solution $y_{\varepsilon}$ obtained by minimizing

$$
J_{\varepsilon}(u)=\left\|A u-f_{\varepsilon}\right\|_{F}^{2}+\varepsilon^{2}\|u\|_{V}^{2}
$$

$\left({ }^{1}\right)$ Manuscrit reçu le 7 juillet 1976, révısıon reçue le 3 janvier 1977

$\left({ }^{2}\right)$ Angewandte Mathematik und Informatık, Fachbereich 10 der Unıversitat der Saarlandes Universitat des Saarlandes, Saarbrucken 
in $V$. It was shown by Tikhonov [10] and Philipps [8] that $y_{\varepsilon} \rightarrow y$ in $E$ if $\varepsilon \rightarrow 0$; see also Ribière [9]. For a more recent exposition, see Céa [2].

As $E$ is of infinite dimension, $y_{\varepsilon}$ has to be computed by some discretization procedure yielding an approximation $y_{\varepsilon h}$ to $y_{\varepsilon}, h$ being the discretization parameter designed to tend to zero. It seems that little attention has been paid to estimates on $\left\|y_{\varepsilon h}-y\right\|_{E}$. Of course $h$ has to be related to $\varepsilon$ somehow if $y_{\varepsilon h}$ is to be as accurate as $y_{\varepsilon}$.

This paper is intended to give a relation of this kind for the finite element method. This relation reads $h=0\left(\varepsilon^{1 / \mu}\right)$ where $\mu$ depends on the finite elements and on the ill-posedness of problem (1.1). If problem (1.1) gets more ill-posed $\mu$ increases. Thus we come to a somewhat surprising conclusion: The more ill-posed the problem is, the coarser the mesh should be chosen.

In $\S 2$, we derive an estimate on $\left\|y-y_{\varepsilon}\right\|_{E}$ similar to the one given by Franklin [3]. In $\S 3$ we give the basic estimate on $\left\|y_{\varepsilon h}-y\right\|_{E}$. In $\S 4$ we apply our result to the numerical inversion of the Radon transform. In $\S 5$ we give numerical results for an integral equation of the first kind.

\section{AN ERROR ESTIMATE FOR THE REGULARIZED SOLUTION}

According to Lions' lemma, (Th. 16.4 of [5]) there is a function $C$ such that

$$
\forall \delta>0 \quad \forall u \in V \quad\|u\|_{E} \leqslant \delta\|u\|_{V}+C(\delta)\|A u\|_{F} .
$$

We assume that $C$ is continuous and non-increasing. Then the function $\delta \rightarrow \delta / C(\delta)$ has an inverse $\gamma$ with $\gamma(\varepsilon) \rightarrow 0$ if $\varepsilon \rightarrow 0$. A typical example is $E=L_{2}(\Omega)$ where $\Omega \subseteq R^{n}, V=H^{k}(\Omega)$, the Sobolev space of order $k>0$, and $\|A u\|_{F}$ equivalent on $V$ to the norm of $H^{-l}(\Omega)=\left(H^{l}(\Omega)\right)^{\prime}$ where $l>0$. It follows from standard interpolation inequalities (see Aziz-Babuska [1], p. 25) that

$$
C(\delta)=\beta \delta^{-l / k}, \quad \text { i.e. } \gamma(\varepsilon)=(\varepsilon / \beta)^{k /(l+k)}
$$

with $\beta$ independent of $\varepsilon$.

THEOREM $1:$ If $y \in V$, then

$$
\left\|y-y_{\varepsilon}\right\|_{E} \leqslant 4\left(1+\|y\|_{V}^{2}\right)^{1 / 2} \gamma(\varepsilon)
$$
from

Proof : Following Tikhonov [10] we start out from $J_{\varepsilon}\left(y_{\varepsilon}\right) \leqslant J_{\varepsilon}(y)$, i.e.

$$
\begin{aligned}
\left\|A y_{\varepsilon}-f_{\varepsilon}\right\|_{F}^{2}+\varepsilon^{2}\left\|y_{\varepsilon}\right\|_{V}^{2} & \leqslant\left\|A y-f_{\varepsilon}\right\|_{F}^{2}+\varepsilon^{2}\|y\|_{V}^{2} \\
& =\left\|f-f_{\varepsilon}\right\|_{F}^{2}+\varepsilon^{2}\|y\|_{V}^{2} \\
& \leqslant \varepsilon^{2}\left(1+\|y\|_{V}^{2}\right) .
\end{aligned}
$$


It follows that

$$
\begin{aligned}
\left\|y_{\varepsilon}\right\|_{V}^{2} & \leqslant 1+\|y\|_{V}^{2}, \\
\left\|A y_{\varepsilon}-f_{\varepsilon}\right\|_{F}^{2} & \leqslant \varepsilon^{2}\left(1+\|y\|_{V}^{2}\right) .
\end{aligned}
$$

The last inequality implies

$$
\begin{aligned}
\left\|A\left(y_{\varepsilon}-y\right)\right\|_{F} & =\left\|A y_{\varepsilon}-f\right\|_{F} \leqslant\left\|A y_{\varepsilon}-f_{\varepsilon}\right\|_{F}+\left\|f_{\varepsilon}-f\right\|_{F} \\
& \leqslant \varepsilon\left\{\left(1+\|y\|_{V}^{2}\right)^{1 / 2}+1\right\} .
\end{aligned}
$$

By (2.1) we get from (2.3), (2.4)

$$
\begin{aligned}
\left\|y-y_{\varepsilon}\right\|_{E} & \leqslant \delta\left\|y-y_{\varepsilon}\right\|_{V}+C(\delta)\left\|A\left(y-y_{\varepsilon}\right)\right\|_{F} \\
& \leqslant \delta\left\{\|y\|_{V}+\left(1+\|y\|_{V}^{2}\right)^{1 / 2}\right\}+\varepsilon C(\delta)\left\{\left(1+\|y\|_{V}^{2}\right)^{1 / 2}+1\right\} \\
& \leqslant 2\left(1+\|y\|_{V}^{2}\right)^{1 / 2}\{\delta+\varepsilon C(\delta)\} .
\end{aligned}
$$

The theorem follows by choosing $\delta=\varepsilon C(\delta)$.

\section{THE FINITE ELEMENT APPROXIMATION TO THE REGULARIZED SOLUTION}

Now let $E=L_{2}(\Omega)$, where $\Omega$ is some region in the euclidean $n$-space. We put $V=H^{k}(\Omega)$, the Sobolev space of order $k>0$. We assume that there are constants $l>0, C_{1}$ such that

$$
\forall u \in V \quad\|A u\|_{F} \leqslant C_{1}\|u\|_{H^{-l}(\Omega)},
$$

where $H^{-l}(\Omega)$ is the dual space of $H^{l}(\Omega)$.

This means that we require a certain amount of ill-posedness, the amount being measured by the number $l$.

The finite element space $S_{h}^{t, k}(\Omega)$ we use is supposed to be a $(t, k)$-regular system which satisfies the inverse assumption (see Aziz-Babuska [1], chapt. 4). 'From theorem 4.1.5 of Aziz-Babuska [1] we conclude that there is a map $I_{h}: H^{k}(\Omega) \rightarrow S_{h}^{t, k}(\Omega)$ such that

$$
\begin{aligned}
& \left\|u-I_{h} u\right\|_{H^{-l}(\Omega)} \leqslant C_{2} h^{\mu}\|u\|_{V}, \\
& \forall u \in I \\
& \left\|I_{h} u\right\|_{V} \leqslant C_{2}\|u\|_{V}
\end{aligned}
$$

where $C_{2}$ is independent of $h$ and

$$
\mu=k+l \text {. }
$$

It is this approximation property of $S_{h}^{t, k}(\Omega)$ with respect to negative norms which will play the essential role in the proof of theorem 2 below.

Naturally, the finite element approximation $y_{\varepsilon h}$ to $y_{\varepsilon}$ is defined by minimizing $J_{\varepsilon}(u)$ in $S_{h}^{t, k}(\Omega)$. Now we are able to state the main result of our paper.

Theorem 2: Let $y \in V$. Assume that there is a constant $C_{3}$ independent of $\varepsilon, h$ such that $h^{\mu} \leqslant C_{3} \varepsilon$. Then there is a constant $C_{4}$, independent on $y, h, \varepsilon$, 
such that

$$
\left\|y_{\varepsilon h}-y\right\|_{E} \leqslant C_{4}\left(1+\|y\|_{V}^{2}\right)^{1 / 2} \gamma(\varepsilon)
$$

Proof : Starting out from $J_{\varepsilon}\left(y_{\varepsilon h}\right) \leqslant J_{\varepsilon}\left(I_{h} y\right)$, we get from (3.1), $\left\|f-f_{\varepsilon}\right\| \leqslant \varepsilon$, (3.2) and from $h^{\mu} \leqslant C_{3} \varepsilon$

$$
\begin{aligned}
\left\|A y_{\varepsilon h}-f_{\varepsilon}\right\|_{F}^{2}+\varepsilon^{2}\left\|y_{\varepsilon h}\right\|_{V}^{2} & \leqslant\left\|A I_{h} y-f_{\varepsilon}\right\|_{F}^{2}+\varepsilon^{2}\left\|I_{h} y\right\|_{V}^{2} \\
& =\left\|A\left(I_{h} y-y\right)+f-f_{\varepsilon}\right\|_{F}^{2}+\varepsilon^{2}\left\|I_{h} y\right\|_{V}^{2} \\
& \leqslant\left(\left\|A\left(I_{h} y-y\right)\right\|_{F}+\left\|f-f_{\varepsilon}\right\|_{F}\right)^{2}+\varepsilon^{2}\left\|I_{h} y\right\|_{V}^{2} \\
& \leqslant\left(C_{1}\left\|I_{h} y-y\right\|_{H^{-l}(\Omega)}+\varepsilon\right)^{2}+\varepsilon^{2}\left\|I_{h} y\right\|_{V}^{2} \\
& \leqslant\left(C_{1} C_{2}\|y\|_{V} h^{\mu}+\varepsilon\right)^{2}+\varepsilon^{2} C_{2}^{2}\|y\|_{V}^{2} \\
& \leqslant\left\{\left(C_{1} C_{2} C_{3}\|y\|_{V}+1\right)^{2}+C_{2}^{2}\|y\|_{V}^{2}\right\} \varepsilon^{2} \\
& \leqslant C_{5}^{2}\left(1+\|y\|_{V}^{2}\right) \varepsilon^{2},
\end{aligned}
$$

where $C_{5}$ independent on $y, h, \varepsilon$. As in the proof of theorem 1 , it follows that

$$
\begin{gathered}
\left\|y_{\varepsilon h}\right\|_{V}^{2} \leqslant C_{5}^{2}\left(1+\|y\|_{V}^{2}\right), \\
\left\|A\left(y_{\varepsilon h}-y\right)\right\|_{F} \leqslant \varepsilon\left\{\left(1+\|y\|_{V}^{2}\right)^{1 / 2} C_{5}+1\right\} .
\end{gathered}
$$

Using again Lions' inequality (2.1) exactly in the same way as in the proof of theorem 1 yields the desired estimate.

\section{APPLICATION TO THE RADON TRANSFORM}

Let $\Omega$ be a bounded domain in $R^{2}$. The Radon transform of $y \varepsilon L_{2}(\Omega)$ is defined to be

$$
(A y)(s, \phi)=\int y(x) d \sigma_{s, \phi}(x)
$$

Here, $s \in R^{1}, 0 \leqslant \phi<2 \pi$, and $\sigma_{s, \phi}$ is the Lebesgue measure on the straight line $x_{1} \cos \phi+x_{2} \sin \phi=s$. We tacitly assume $y$ to be extended to the whole of $R^{2}$ by putting $y=0$ outside of $\Omega$.

Let $Z \subseteq R^{3}$ be the cylinder $\left\{(\cos \phi, \sin \phi, s): 0 \leqslant \phi<2 \pi, s \in R^{1}\right\}$.

In $L_{2}(Z)$ we introduce the norm

$$
\|f\|_{L_{2}(z)}^{2}=\int_{0}^{2 \pi} \int_{-\infty}^{+\infty} f^{2}(\cos \phi, \sin \phi, s) d \phi d s .
$$

It follows from the work of Ludwig [6] that $A$ may be viewed as a map from $L_{2}(\Omega)$ into $L_{2}(Z)$. Moreover, we have.

LeMma 4.1 : The norms $\|A y\|_{L_{2}(Z)},\|y\|_{H^{-1^{\prime \prime}(\Omega)}}$ are equivalent on $L_{2}(\Omega)$. 
Proof : Following Ludwig [6]. p. 51 we write

$$
(A y)(s, \phi)=\int_{-\infty}^{+\infty} \mathrm{e}^{i s r} \tilde{y}(r \cos \phi, r \sin \phi) d r
$$

where

$$
\tilde{y}(\xi)=\frac{1}{2 \pi} \int_{R^{2}} \mathrm{e}^{-i x \xi} y(y) d x
$$

is the Fourier transform of $y$. Applying Parseval's relation to (4.1) we obtain

$$
\int_{-\infty}^{+\infty}((A y)(s, \phi))^{2} d s=2 \pi \int_{-\infty}^{+\infty}|\tilde{y}(r \cos \phi, r \sin \phi)|^{2} d r .
$$

Integrating with respect to $\phi$ we get

$$
\begin{aligned}
\|A y\|_{L_{2}(Z)}^{2} & =2 \pi \int_{0}^{2 \pi} \int_{-\infty}^{+\infty}|\tilde{y}(r \cos \phi, r \sin \phi)|^{2} d r d \phi \\
& =2 \pi \int_{R^{2}}|\xi|^{-1}|\tilde{y}(\xi)|^{2} d \xi .
\end{aligned}
$$

Using the norm

$$
\|y\|_{H^{-1 / 2}\left(R^{2}\right)}^{2}=\int_{R^{2}}\left(1+|\xi|^{2}\right)^{-1 / 2}|\tilde{y}(\xi)|^{2} d \xi
$$

in $H^{-1 / 2}\left(R^{2}\right)$, it follows from (4.2) and supp $(y) \subseteq \bar{\Omega}$ that $\|A y\|_{L_{2}(Z)}$ and $\|y\|_{H^{-1 / 2}\left(R^{2}\right)}$ are equivalent. Here we used the fact that the norms $\|y\|_{H^{-1 / 2}\left(R^{2}\right)}$ and $\|y\|_{H^{-1 / 2}(\Omega)}$ are equivalent. This follows from the extension theorem 2.3.2 and the definition 2.3.4 of the norm in $H^{1 / 2}$ of [1]. Thus the lemma is proved.

Now it is easy to apply the results of our paper to the Radon transform : We put $E=L_{2}(\Omega), F=L_{2}(Z), V=H^{1}(\Omega)$. From (2.2) we get that $C(\delta)=0\left(\delta^{-1 / 2}\right)$ in $(2.1)$, hence $\gamma(\varepsilon)=0\left(\varepsilon^{2 / 3}\right)$. Thus, theorem 1 states that

$$
\left\|y-y_{\varepsilon}\right\|_{L^{2}(\Omega)}=0\left(\varepsilon^{2 / 3}\right) \text {. }
$$

Let $\Omega$ be a square region and $\left(T_{h}\right)$ a regular sequence of triangulations of $\Omega$ i.e. there is $C \geqslant 1$ such that each triangle of $T_{h}$ contains a ball of radius $h / C$ ans is contained in a ball of radius $C h$. Then

$$
S_{h}^{2,1}(\Omega)=\left\{u \in C(\Omega): u \text { linear in each triangle of } T_{h}\right\}
$$

is a $(2,1)$-regular system. Theorem 2 tells us that it suffices to use a mesh size $h=0\left(\varepsilon^{2 / 3}\right)$ in order to obtain the estimate

$$
\left\|y-y_{\varepsilon h}\right\|_{L_{2}(\Omega)}=0\left(\varepsilon^{2 / 3}\right) \text {. }
$$


It is doubtful (at best!) wether this method can compete with older ones as described e.g. in Guenther et al. [4], by the following reasons : It is not clear wether an efficient implementation is possible, and, more seriously, the assumption that $y \in H^{1}(\Omega)$ is not realistic for many application e.g. in computerized tomography. Nevertheless, it seems that presently this finite element method is the only one the convergence of which is on a sound theoretical basis.

\section{APPLICATION TO INTEGRAL EQUATIONS OF THE FIRST KIND}

Let $E=L_{2}(a, b), F=L_{2}(c, d)$ and for $y \in E$

$$
(A y)(x)=\int_{a}^{b} K(x, t) y(t) d t
$$

where $K \in C^{\infty}([a, b] \times[c, d])$. Then the norm $\|A y\|_{F}$ is weaker then the norm of $H^{-l}(a, b)$ for any finite $l$. Thus if $V=H^{k}(a, b), k>0$, then $C(\delta) \rightarrow \infty$ in (2.1) for $\delta \rightarrow 0$ faster than any power of $\delta$, i.e. $\gamma(\varepsilon) \rightarrow 0$ for $\varepsilon \rightarrow 0$ slower than any power of 2 . Consequently, the accuracy of $y_{\varepsilon}$ guaranteed by theorem 1 is very poor. This result explains the difficulties one encounters in the numerical solution of the Fredholm integral equation of the first kind $A y=f$.

One might guess that a very accurate discretization procedure is needed to deal with a problem which is ill-posed that much. Theorem 2 tells us that the opposite is true. If we use for $S_{h}^{k+1, k}$ splines of class $C^{k-1}$ and of degree $k$ on a uniform mesh with mesh size $h$, then, according to theorem 2, $h=\varepsilon^{1 / 2(k+l)}$ is an appropriate choice for $h$. As $l$ can be chosen arbitrarily large, we can keep $h$ nearly constant if $\varepsilon \rightarrow 0$.

To see what this means in practice we solved

$$
\int_{0}^{1} \exp \left(-5(x-t)^{2}\right) y(t) d t=f(x), \quad 0 \leqslant x \leqslant 1
$$

where $y(t)=\exp (-t)$. We considered the case $k=1$.

The right hand side $f$ was given exactly. The numerical results are given in table 1. Smaller values of $h$ than those noted in table 1 gave no essential decrease or even a slight increase of the error. Thus the optimal value of $h$ was always in $[1,1 / 4]$ while $\varepsilon$ varied from $10^{-2}$ to $10^{-5}$. This clearly coincides with our theory.

As a second example we solved the problem

$$
\int_{0}^{1}(\sinh ((t-1) x))^{2} y(t) d t=f(x), \quad 0 \leqslant x \leqslant 3
$$


which arises in finding the dielectric profile of a slab (see [5], p. 148). The exact solution was taken to be $y(t)=\exp (-t)$. The computation, the results of which are shown in table 2 , was carried out with $k=2$.

TABLE 1

$\begin{array}{clc}\varepsilon & h & \left\|y-y_{\varepsilon h}\right\|_{L_{2}(0,1)} \\ 10^{-2} & 1 & 0.31 \\ & 1 / 2 & 0.31 \\ & 1 / 3 & 0.31 \\ 10^{-3} & 1 / 4 & 0.31 \\ & 1 & 0.31 \\ & 1 / 2 & 0.30 \\ 10^{-4} & 1 / 3 & 0.30 \\ & 1 / 4 & 0.30 \\ & 1 & 0.31 \\ 10^{-5} & 1 / 2 & 0.085 \\ & 1 / 3 & 0.075 \\ & 1 / 4 & 0.072 \\ & 1 & 0.31 \\ & 1 / 2 & 0.065 \\ & 1 / 3 & 0.035 \\ & 1 / 4 & 0.046\end{array}$

TABLE 2

$\begin{array}{cc}\varepsilon & h \\ 10^{-1} & 1 \\ & 1 / 2 \\ 10^{-2} & 1 / 3 \\ & 1 \\ & 1 / 2 \\ 10^{-3} & 1 / 3 \\ & 1 \\ & 1 / 2 \\ 10^{-4} & 1 / 3 \\ & 1 \\ & 1 / 2 \\ & 1 / 3\end{array}$

$\left\|y-y_{\varepsilon h}\right\|_{L_{2}(0,1)}$

0.14

0.14

0.14

0.061

0.061

0.061

0.048

0.048

0.047

0.0048

0.011

0.0067

Again we see that for all realistic values of $\varepsilon$, a very modest step size suffices. The strange behavior of the error for $\varepsilon=10^{-4}$ is related to the vanishing of $K(x, t)$ at $t=1$ which is responsible for very large errors of $y_{\varepsilon}$ near $t=1$. For $h=1, y_{\varepsilon h}$ is a quadratic on [0,1] approximating $y_{\varepsilon}$ in an $L_{2}$-sence. Thus $y_{\varepsilon h}$ is not likely to exhibit the same growth of error near $t=1$ as does $y_{\varepsilon}$. For $h=1 / 2,1 / 3, y_{\varepsilon h}$ is a spline which can be fitted much easier to $y_{\varepsilon}$ even for $t$ close to 1 . This argument is supported by the numerical results which give an error of $-0.0181,0.0358$ and 0.0251 for $y_{\varepsilon h}(1)$ if $h=1,1 / 2,1 / 3$, respectively. 
I would like to thank Dr. Edenhofer, Oberpfaffenhofen, and Prof. R. B. Guenther, Corvallis, for providing me with valuable references.

\section{REFERENCES}

1. A. K. AzIz and I. BabuSKa, Survey Lectures on the Mathematical Foundations of the Finite Element Method. In : Azız, A. K. (ed.) : The Mathematical Foundations of the Finite Element Method with Application to Partial Differential Equations, Academic Press, 1972.

2. I. Cea, Optimisation, Théorie et Algorithmes. Dunod, Paris, 1971.

3. J. N. Franklin. On Tikhonov's Method for Ill-Posed Problems. Math. Comp. 28. 1974, p. $889-907$

4. R. B. Guenther, C. W. Kerber, E. K. Killian, K. T. Smith and S. L. Wagner, Reconstruction of objects form Radiographs and the Location of Brain Tumors. Proc. Nat. Acad. Sci. USA. 71, 1974 p. 4884-4886.

5. I. L. Lions et E. MAGENES, Problème avec limites non homogènes et applications, vol. 1. Dunod, Paris, 1968.

6. D. Ludwig, The Radon Transform on Euclidean Space. Comm. Pure Applied Math. $19,1966,49-81$.

7. R. Mrtrra and C. A. KLEIN, Stability and Convergence of Moment Method Solutions. In : MitTrA, R. (ed) : Numerical and Asymptotic Techniques in Electromagnetics, Spinger, 1975.

8. D. L. Philipps. A Technique for the Numerical Solution of Certain rntegral Equations of the First Kind. J. Ass. Comp. Mach. 9, 1962, p. 84-97.

9. G. RIBIÈre, Régularisation d'opérateurs. R.I.R.O., 1, 1967, p. 57-79.

10. A. N. Tiknonov, The Regularization of Incorrectly Posed Problems. Dokl. Akad. Nauk SSSR, 153, 1963, p. 42-52. 